\title{
SINGULAR PERTURBATIONS ON THE INFINITE INTERVAL
}

\author{
BY \\ FRANK CHARLES HOPPENSTEADT( $\left.{ }^{1}\right)$
}

Initial-value problems of the form

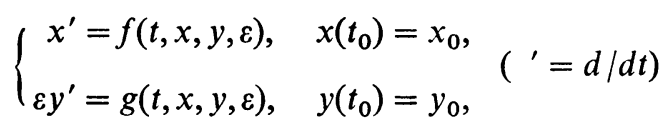

where $\varepsilon$ is a small positive parameter arise frequently in applications. Here $f$ and $x$ are real $k$-dimensional vectors with components $f=\left(f_{1}, \cdots, f_{k}\right)$ and $x=\left(x_{1}, \cdots, x_{k}\right)$, respectively, and $g$ and $y$ are real $j$-dimensional vectors with components $g$ $=\left(g_{1}, \cdots, g_{j}\right)$ and $y=\left(y_{1}, \cdots, y_{j}\right)$, respectively. The purpose of this paper is to investigate the behavior of solutions of $\left(P_{\varepsilon}\right)$ as $\varepsilon \rightarrow 0^{+}$for $t_{0} \leqq t<\infty$.

In studying the behavior of solutions of $\left(P_{\varepsilon}\right)$ for small positive $\varepsilon$ it is convenient to make use of two systems which are associated with $\left(P_{\varepsilon}\right)$. The first associated system, called the degenerate system, is obtained by formally setting $\varepsilon=0$ in $\left(P_{\varepsilon}\right)$ : This gives

$\left(P_{0}\right)$

$$
\left\{\begin{array}{l}
x=f(t, x, y, 0), \quad x\left(t_{0}\right)=x_{0}, \\
0=g(t, x, y, 0) .
\end{array}\right.
$$

The second associated system is obtained by making the "stretching" transformation of independent variable $s=(t-\alpha) / \varepsilon$ in $\left(P_{\varepsilon}\right)$ and then setting $\varepsilon=0$ in the result: This gives

$$
\begin{aligned}
& d x / d s=0, \\
& d y / d s=g(\alpha, x, y, 0) .
\end{aligned}
$$

Since the only solution of $d x / d s=0$ is $x=\beta=$ constant, this system can be written in the more convenient form

$$
d y / d s=g(\alpha, \beta, y, 0), \quad y(0)=y_{0}
$$

Presented to the Society, April 10, 1965; received by the editors October 4, 1965 and, in revised form, January 13, 1966.

(1) Part of a dissertation submitted to the University of Wisconsin for the degree of Doctor of Philosophy. The author wishes to thank Professor F. G. Brauer for his encouragement and guidance. This work was partially supported by the U.S. Army Research Office, Durham, North Carolina, Grant DA-31-124-ARO-D-268. 
where $(\alpha, \beta)$ are treated as parameters. The system $(B L)$ is called the boundarylayer system.

In this paper conditions on the functions $f$ and $g$ are found under which the solution of $\left(P_{\varepsilon}\right)$ for small $\varepsilon$ approximates the solution of $\left(P_{0}\right)$ on the interval $t_{0}<t<\infty$.

The work presented in [3], [4], [5] and [8] deals with this problem when $t$ is restricted to compact intervals. In order to obtain results these authors impose various stability conditions on the boundary-layer system (BL). In the papers [3], [4] and [5] the stability conditions take the form of conditions on the eigenvalues of the coefficient matrix of a certain variational system associated with (BL). In [8] A. N. Tihonov requires that a certain solution of (BL) be asymptotically stable in the sense of Liapunov.

Our treatment of the case when $t$ is allowed to range over the entire positive real axis requires that both the associated systems $\left(P_{0}\right)$ and $(B L)$ satisfy severe stability conditions. However, these conditions reduce to those needed by Tihonov when compact $t$ intervals are considered.

The result of this paper is the best possible in the sense that the hypotheses cannot be substantially weakened. A series of examples accompanying the theorem investigates the possibility of altering the hypotheses.

1. Preliminaries. In what follows the norm of a vector (or matrix) is taken to be the sum of the absolute values of the components of that vector (or matrix); e.g., $|x|=\Sigma\left|x_{i}\right|$. Let $I=[0, \infty), S_{R}=\left\{(x, y) \in E^{k+j}:|x|+|y| \leqq R\right\}$, and let $S_{R \mid x}$ and $S_{R \mid y}$ represent the restriction of $S_{R}$ to $E^{k}$ and $E^{j}$, respectively.

Let us assume that the functions $f$ and $g$ satisfy the following conditions.

(I) The system $\left(P_{0}\right)$ has a solution $x=x(t), y=y(t)$ which exists for $t_{0} \leqq t<\infty$.

With assumption (I), an obvious transformation takes system $\left(P_{0}\right)$ into an equivalent system which has $x=0, y=0$ as a solution. It is convenient to assume henceforth that system $\left(P_{0}\right)$ has $x=0, y=0$ as a solution for all $t_{0} \leqq t<\infty$.

(II) $f, g, f_{x}, f_{y}, g_{t}, g_{x}, g_{y} \in C\left(I \times S_{R} \times\left[0, \varepsilon_{0}\right]\right)$. Here $f_{x}$ denotes the matrix with components $\left(\partial f_{i} / \partial x_{l}\right), i, l=1, \cdots, k$, and similarly for $f_{y}, g_{x}$ and $g_{y}$.

(III) There exists a bounded, twice continuously differentiable function $y=Y(t, x)$ such that

$$
g(t, x, Y(t, x), 0)=0 \text { for all }(t, x) \in I \times S_{R \mid x} .
$$

Furthermore, $Y(t, x)$ is isolated in the sense that $z \neq Y\left(t_{1}, x_{1}\right)$ and $g\left(t_{1}, x_{1}, z, 0\right)=0$ for some $\left(t_{1}, x_{1}\right) \in I \times S_{R \mid x}$ imply $\left|z-Y\left(t_{1}, x_{1}\right)\right|>R$.

With assumption (III) there is no loss of generality in assuming that $Y(t, x)=0$ for all $(t, x) \in I \times S_{R \mid x}$. Indeed, if this is not already the case, the transformation

$$
\begin{aligned}
& x=w, \\
& y=z+Y(t, w)
\end{aligned}
$$


takes system $\left(P_{\varepsilon}\right)$ into

$$
\begin{aligned}
w^{\prime} & =f(t, w, z+Y(t, w), \varepsilon)=F(t, w, z, \varepsilon), \\
\varepsilon z^{\prime} & =g(t, w, z+Y(t, w), \varepsilon)-\varepsilon\left[Y_{t}(t, w)+Y_{x}(t, w) \cdot f(t, w, z+Y(t, w), \varepsilon)\right] \\
& =G(t, w, z, \varepsilon),
\end{aligned}
$$

which is of the same form as system $\left(P_{\varepsilon}\right)$ with $G(t, w, 0,0)=0$ for all $(t, w) \in I \times S_{R \mid x}$.

With assumption (III) the degenerate system can be written in the more convenient form

$$
x^{\prime}=f(t, x, 0,0), \quad x\left(t_{0}\right)=x_{0} .
$$

The following four hypotheses are the crucial ones.

(IV) The function $f$ is continuous at $y=0, \varepsilon=0$ uniformly in $(t, x) \in I \times S_{R \mid x}$, and $f(t, x, 0,0)$ and $f_{x}(t, x, 0,0)$ are bounded on $I \times S_{R \mid x}$.

(V) The function $g$ is continuous at $\varepsilon=0$ uniformly in $(t, x, y) \in I \times S_{R}$, and $g(t, x, y, 0)$ and its derivatives with respect to $t$ and the components of $x$ and $y$ are bounded on $I \times S_{R}$.

The last two assumptions are the stability properties required of the two associated systems. To simplify the explanations, let $\mathscr{K}$ be the class of all continuous, strictly increasing, real-valued functions $d(r), 0 \leqq r$, with $d(0)=0$; and, let $\mathscr{S}$ be the class of all nonnegative, strictly decreasing, continuous, real-valued functions $\sigma(s), 0 \leqq s<\infty$, for which $\sigma(s) \rightarrow 0$ as $s \rightarrow \infty$.

(VI) The zero solution of (D) is uniform-asymptotically stable. That is, if $x=\Phi\left(t, t_{0}, x_{0}\right)$ is the solution of (D), there exist $d \in \mathscr{K}$ and $\sigma \in \mathscr{S}$ such that

$$
\left|\Phi\left(t, t_{0}, x_{0}\right)\right| \leqq d\left(\left|x_{0}\right|\right) \sigma\left(t-t_{0}\right) \text { for }\left|x_{0}\right| \leqq R \text { and } 0 \leqq t_{0} \leqq t<\infty
$$

(cf. Hahn [6,p. 18]).

It is now convenient to define a new form of stability.

(VII) The zero solution of (BL) is uniform-asymptotically stable uniformly in the parameters $(\alpha, \beta) \in I \times S_{R \mid x}$. That is, if $y=\Psi\left(s, y_{0}, \alpha, \beta\right)$ is the solution of (BL), there exist $e \in \mathscr{K}$ and $\rho \in \mathscr{S}$ such that

$$
\left|\Psi\left(s, y_{0}, \alpha, \beta\right)\right| \leqq e\left(\left|y_{0}\right|\right) \rho(s)
$$

for all $0 \leqq s<\infty,\left|y_{0}\right| \leqq R$ and $(\alpha, \beta) \in I \times S_{R \mid x}$.

The main result of this paper is then:

THEOREM. Let the conditions (I) through (VII) be satisfied. Then for sufficiently small $\left|x_{0}\right|+\left|y_{0}\right|$ and $\varepsilon$ the solution of the perturbed system $\left(P_{\varepsilon}\right)$ exists for $t_{0} \leqq t<\infty$, and this solution converges to the solution of the degenerate system $\left(P_{0}\right)$ as $\varepsilon \rightarrow 0^{+}$uniformly on all closed subsets of $t_{0}<t<\infty$. 
The essential difference between this result and all previous results is that the $t$-sets on which the uniform convergence occurs are closed but not necessarily bounded. In particular, sets of the form $t_{0}<t_{1} \leqq t<\infty$ are included.

2. Examples. The significance of the hypotheses in the above theorem can best be understood by considering examples of systems of the form $\left(P_{\varepsilon}\right)$. The first four examples are concerned with the possibility of weakening any of the hypotheses (IV)-(VII) in the above theorem. In each of the examples E.1-E.4 one of the conditions (IV)-(VII) is weakened, and a system of the form $\left(P_{\varepsilon}\right)$ is presented which satisfies the altered hypotheses. In each case there are solutions beginning arbitrarily near $x=0, y=0$ for which the convergence in the theorem is not uniform on sets of the form $t_{0}<t_{1} \leqq t<\infty$.

The last example, E.5, shows that in the case where $f$ and $g$ are linear in the components of $x$ and $y$, it is not possible to replace condition (VI) by a restriction on the eigenvalues of the coefficient matrix of the degenerate system. Such an approach was attempted by V. Butuzov [1].

E.1. Let condition (IV) be replaced by

$\left(\mathrm{IV}^{\prime}\right) f$ is continuous and bounded on $I \times S_{R} \times\left[0, \varepsilon_{0}\right]$.

The system

$$
\begin{cases}x^{\prime}=(-1+\sin \varepsilon t) x, & x(0)=x_{0}, \\ \varepsilon y^{\prime}=-y, & y(0)=y_{0}\end{cases}
$$

satisfies the hypotheses of the theorem with condition (IV) replaced by (IV'). Here $f(t, x, y, \varepsilon)=(-1+\sin \varepsilon t) x$ is clearly not continuous at $\varepsilon=0$ uniformly in $t \quad 0 \leqq t<\infty$. The solution of (1) is

$$
\begin{aligned}
& x=x_{0} \exp \{-t+(1-\cos \varepsilon t) / \varepsilon\} \\
& y=y_{0} \exp (-t / \varepsilon) .
\end{aligned}
$$

The degenerate system associated with (1) $(\varepsilon=0$ in (1)) is

$$
\left\{\begin{array}{l}
x^{\prime}=-x, \quad x(0)=x_{0} \\
y=0
\end{array}\right.
$$

which has as a solution

$$
\begin{aligned}
& x=x_{0} \exp (-t), \\
& y=0
\end{aligned}
$$

Since $(1-\cos \varepsilon t) / \varepsilon$ does not converge to zero as $\varepsilon \rightarrow 0^{+}$uniformly on any unbounded $t$-interval, the solution of (1) does not converge to the solution of (2) as $\varepsilon \rightarrow 0^{+}$uniformly on any set of the form $0<t_{1} \leqq t<\infty$. 
E.2. Let condition (V) be replaced by

$\left(V^{\prime}\right) g$ and the partial derivatives of $g$ with respect to $t$ and the components of $x$ and $y$ are bounded on $I \times S_{R} \times\left[0, \varepsilon_{0}\right]$.

The system

$$
\left\{\begin{aligned}
x^{\prime} & =-x, & x(0) & =x_{0} \\
\varepsilon y^{\prime} & =-\cos (\varepsilon t) y, & y(0) & =y_{0}
\end{aligned}\right.
$$

satisfies the hypotheses of the theorem with condition $(V)$ replaced by $\left(V^{\prime}\right)$ and has as a solution

$$
\begin{aligned}
& x=x_{0} \exp (-t), \\
& y=y_{0} \exp \left(-\sin \varepsilon t / \varepsilon^{2}\right) .
\end{aligned}
$$

The degenerate system associated with (3) is

$$
\left\{\begin{array}{l}
x^{\prime}=-x, \quad x(0)=x_{0} \\
y=0
\end{array}\right.
$$

whose solution is

$$
\begin{aligned}
& x=x_{0} \exp (-t), \\
& y=0 .
\end{aligned}
$$

Even though $\lim _{\varepsilon \rightarrow 0^{+}} \exp \left(-\sin \varepsilon t / \varepsilon^{2}\right)=0$ for each $t>0$, this limit is not uniform in $t$ on any set of the form $0<t_{1} \leqq t<\infty$. Hence, the solution of (3) does not converge to the solution of (4) as $\varepsilon \rightarrow 0^{+}$uniformly on sets of the form $0<t_{1} \leqq t<\infty$.

E.3. Let condition (VI) be replaced by

(VI') The zero solution of the degenerate system is asymptotically stable.

We first observe that the zero solution of

$$
x^{\prime}=-x / t, \quad x\left(t_{0}\right)=x_{0} \quad\left(t_{0} \geqq 1\right)
$$

is asymptotically stable but not uniform-asymptotically stable. The solutions are of the form $x=x_{0} t_{0} / t$, and so do not tend to zero as $t \rightarrow \infty$ uniformly in $t_{0}$. On the other hand, the zero solution of $y^{\prime}=-y^{3} / 2$ is uniform-asymptotically stable. The solution, $y=y_{0} /\left[1+y_{0}^{2}\left(t-t_{0}\right)\right]^{1 / 2}$ satisfies the inequality

$$
\left|y_{0} /\left[1+y_{0}^{2}\left(t-t_{0}\right)\right]^{1 / 2}\right| \leqq\left.|| y_{0}\right|^{1 / 2} /\left(t-t_{0}\right)^{1 / 4} \mid \quad \text { for } t \geqq t_{0} .
$$

With this we see that the system

$$
\left\{\begin{aligned}
x^{\prime} & =-x /(t+\varepsilon)+y, & x\left(t_{0}\right) & =x_{0}, \\
\varepsilon y^{\prime} & =-y^{3} / 2, & y\left(t_{0}\right) & =y_{0}
\end{aligned} \quad\left(t_{0} \geqq 1\right)\right.
$$


satisfies the hypotheses of the theorem with (VI) replaced by (VI'). Using the variation of constants formula, the solution of (5) is

$$
\begin{aligned}
& x=x_{0} t_{0} / t+\left(\varepsilon^{1 / 2} y_{0} / t\right) \int_{t_{0}}^{t}\left[s /\left(\varepsilon+y_{0}^{2}\left(s-t_{0}\right)\right)^{1 / 2}\right] d s, \\
& y=\varepsilon^{1 / 2} y_{0} /\left(\varepsilon+y_{0}^{2}\left(t-t_{0}\right)\right)^{1 / 2} .
\end{aligned}
$$

The degenerate system associated with (5) is

$$
\left\{\begin{array}{l}
x^{\prime}=-x / t+y, \quad x\left(t_{0}\right)=x_{0}, \\
y=0,
\end{array}\right.
$$

which has as a solution

$$
\begin{aligned}
& x=x_{0} t_{0} / t, \\
& y=0 .
\end{aligned}
$$

For $y_{0} \neq 0$, the $x$-component of the solution of (5) is not bounded on any set of the form $1 \leqq t_{1} \leqq t<\infty$. Because of this, the solution of (5) does not converge to the solution of (6) as $\varepsilon \rightarrow 0^{+}$uniformly on any $t$-interval of the form $t_{0}<t_{1} \leqq t<\infty$.

E.4. Let condition (VII) be replaced by

(VII') The zero solution of the boundary-layer system is uniform-asymptotically stable for each $(\alpha, \beta) \in I x S_{R \mid x}$.

First, we shall see that the system

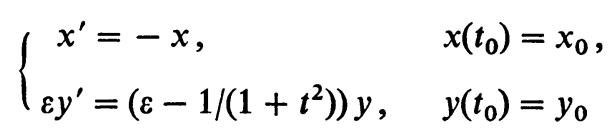

satisfies condition (VII') but not condition (VII). The boundary-layer system associated with (7) is

$$
d y / d s=-y /\left(1+\alpha^{2}\right), \quad y(0)=y_{0}
$$

which has as a solution $y=y_{0} \exp \left(-s /\left(1+\alpha^{2}\right)\right)$. Clearly, $\exp \left(-s /\left(1+\alpha^{2}\right)\right)$ does not tend to zero as $s \rightarrow \infty$ uniformly in $\alpha$ for $0 \leqq \alpha<\infty$. However, for each $\alpha, 0 \leqq \alpha<\infty$, the zero solution of (8) is uniform-asymptotically stable.

System (7) satisfies conditions (I) -(VI) and has a solution

$$
\begin{aligned}
& x=x_{0} \exp \left(t_{0}-t\right), \\
& y=y_{0} \exp \left\{\left(\tan ^{-1} t_{0}-\tan ^{-1} t\right) / \varepsilon\right\} \exp \left(t-t_{0}\right) .
\end{aligned}
$$

The degenerate system associated with (7) is

$$
\left\{\begin{array}{l}
x^{\prime}=-x, \quad x\left(t_{0}\right)=x_{0}, \\
y=0,
\end{array}\right.
$$


which has as a solution

$$
\begin{aligned}
& x=x_{0} \exp \left(t_{0}-t\right), \\
& y=0 .
\end{aligned}
$$

The $y$-component of the solution of (7) is unbounded, and therefore, does not tend to zero as $\varepsilon \rightarrow 0^{+}$uniformly on any interval of the form $t_{0}<t_{1} \leqq t<\infty$.

E.5. Finally, consider the case where $f$ and $g$ are linear in $x$ and $y$ and independent of $\varepsilon$. A system of this form is

$$
\begin{aligned}
x^{\prime} & =B(t) x+C(t) y, & x\left(t_{0}\right) & =x_{0}, \\
\varepsilon y^{\prime} & =D(t) y, & y\left(t_{0}\right) & =y_{0},
\end{aligned}
$$

where $B, C$ and $D$ are matrices of appropriate dimensions. Condition (VI) in this case requires that the zero solution of

$$
x^{\prime}=B(t) x, \quad x\left(t_{0}\right)=x_{0}
$$

be uniform-asymptotically stable. V. Butuzov [1] states that the above theorem is still valid when condition (VI) is replaced by

$\left(\mathrm{VI}^{\prime \prime}\right)$ The eigenvalues $\lambda_{i}(t), \quad i=1, \cdots, k$, of $B(t)$ satisfy $\operatorname{Re}\left(\lambda_{i}(t)\right) \leqq-m<0$ for all $t_{0} \leqq t<\infty$ and some $m>0$.

The example presented below uses the fact that condition ( $\left.\mathrm{VI}^{\prime \prime}\right)$ does not necessarily imply the stability of the zero solution of $(10)$ to show that the uniform convergence of the theorem need not follow if (VI) is replaced by $\left(\mathrm{VI}^{\prime \prime}\right)$.

Let us take $B(t)=U^{-1}(t) A U(t)$ where

$$
U(t)=\left(\begin{array}{rr}
\cos t & \sin t \\
-\sin t & \cos t
\end{array}\right) \text { and } A=\left(\begin{array}{r}
-1-5 \\
0-1
\end{array}\right)
$$

$C(t)=\operatorname{col}(1,1)$, and $D(t)=(-1)$. In this case we have

$$
\begin{aligned}
\left(\begin{array}{l}
x_{1} \\
x_{2}
\end{array}\right)^{\prime} & =B(t)\left(\begin{array}{l}
x_{1} \\
x_{2}
\end{array}\right)+\left(\begin{array}{l}
1 \\
1
\end{array}\right) y, \\
\varepsilon y^{\prime} & =(-1) y .
\end{aligned}
$$

The solution of this system which satisfies the initial conditions $x_{1}(0)=x_{2}(0)$ $=0, y(0)=y_{0}$ is

where

$$
\begin{aligned}
\left(\begin{array}{c}
x_{1}(t, \varepsilon) \\
x_{2}(t, \varepsilon)
\end{array}\right) & =y_{0} \Phi(t) \int_{0}^{t} \Phi^{-1}(s)\left(\begin{array}{c}
e^{-s / \varepsilon} \\
e^{-s / \varepsilon}
\end{array}\right) d s, \\
y(t, \varepsilon) & =y_{0} \exp (-t / \varepsilon)
\end{aligned}
$$

$$
\Phi(t)=\left(\begin{array}{cc}
e^{t}[\cos t+(\sin t) / 2] & e^{-3 t}[\cos t-(\sin t) / 2] \\
e^{t}[\sin t-(\cos t) / 2] & e^{-3 t}[\sin t+(\cos t) / 2]
\end{array}\right)
$$


is a fundamental matrix for

$$
\left(\begin{array}{l}
x_{1} \\
x_{2}
\end{array}\right)^{\prime}=U^{-1}(t) A U(t)\left(\begin{array}{l}
x_{1} \\
x_{2}
\end{array}\right) .
$$

The associated degenerate system is

$$
\begin{aligned}
\left(\begin{array}{l}
x_{1} \\
x_{2}
\end{array}\right)^{\prime} & =B(t)\left(\begin{array}{l}
x_{1} \\
x_{2}
\end{array}\right)+\left(\begin{array}{l}
1 \\
1
\end{array}\right) y, \quad \begin{array}{l}
x_{1}(0)=0, \\
x_{2}(0)=0,
\end{array} \\
y & =0
\end{aligned}
$$

whose solution is $x_{1}=x_{2}=y=0$. A straightforward but tedious calculation shows that

$$
x_{1}(t, \varepsilon)=c(\varepsilon) y_{0} e^{t}(\cos t+(\sin t) / 2)+O(\exp (-t / \varepsilon)) \text { as } t \rightarrow \infty
$$

where $c(\varepsilon) \rightarrow 0$ as $\varepsilon \rightarrow 0^{+}$. From this it is clear that $x_{1}(t, \varepsilon)$ does not tend to 0 as $\varepsilon \rightarrow 0^{+}$uniformly on $0<t_{1} \leqq t<\infty$.

3. Proof of the theorem. First, the stability conditions (VI) and (VII) are used to construct Liapunov functions for the associated systems (D) and (BL), respectively, (Lemmas 1 and 2). These functions are in turn used to define "tubes" in $I \times S_{R}$ which are invariant with respect to the solutions of $\left(P_{\varepsilon}\right)$ for sufficiently small $\varepsilon$, (Lemma 3). Finally, an argument similar to Tihonov's [8] (Lemma 4 here) shows that for sufficiently small $\varepsilon$ the solution of $\left(P_{\varepsilon}\right)$ approximates the solution of $\left(P_{0}\right)$ uniformly on any given compact subset of $\left(t_{0}, \infty\right)$.These results are then combined to establish the uniform approximation of the solution of $\left(P_{0}\right)$ by the solution of $\left(P_{\varepsilon}\right)$ for small $\varepsilon$ on sets of the form $t_{0}<t_{1} \leqq t<\infty$.

To fix ideas let us state conditions (IV) and (V) precisely. The uniform continuity of the function $f$ implies the existence of a function $v(|y|, \varepsilon)$ such that $v(|y|, \varepsilon) \rightarrow 0$ as $|y|+\varepsilon \rightarrow 0$ and $|f(t, x, y, \varepsilon)-f(t, x, 0,0)| \leqq v(|y|, \varepsilon)$ for $(t, x, y) \in I \times S_{R}$ and $0 \leqq \varepsilon \leqq \varepsilon_{0}$. Also, the uniform continuity of $g$ implies the the existence of a function $\mu(\varepsilon)$ such that $\mu(\varepsilon) \rightarrow 0$ as $\varepsilon \rightarrow 0^{+}$and $\mid g(t, x, y, \varepsilon)$ $-g(t, x, y, 0) \mid \leqq \mu(\varepsilon)$ for $(t, x, y) \in I \times S_{R}$ and $0 \leqq \varepsilon \leqq \varepsilon_{0}$.

The construction of the Liapunov functions for the systems (D) and (BL) is accomplished by making use of the following lemma due to J. L. Massera [7] which we state here without proof.

LEMMA 1. Given any $c \in \mathscr{K}, \lambda \in \mathscr{S}$ and any positive, nondecreasing function $N(s), s \geqq 0$, there exists a function $G(r)$ with the following properties:

(i) $G$ and $G^{\prime}$ are continuous, strictly increasing functions for $r \geqq 0$ with $G(0)=G^{\prime}(0)=0$;

(ii) The integrals $\int_{0}^{\infty} G(u(s)) d s$ and $\int_{0}^{\infty} G^{\prime}(u(s)) N(s) d s$ converge for $0 \leqq u(s)$ $\leqq c(R) \lambda(s)$ uniformly in $u$. 
Let $\left\|\Phi\left(t, t_{0}, x_{0}\right)\right\|$ denote the euclidean norm of the solution of (D). The boundedness of the matrix $f_{x}(t, x, 0,0)$ on $I \times S_{R \mid x}$ implies that the derivatives of $\left\|\Phi\left(t, t_{0}, x\right)\right\|$ with respect to the components of $x$ are bounded by a function of the form $K \exp K\left(t-t_{0}\right)$ for $t \geqq t_{0}$ where $K$ is a constant independent of $t, t_{0}$, and $x$.

If we take for $c$ and $\lambda$ in Massera's lemma $d \in \mathscr{K}$ and $\sigma \in \mathscr{S}$ given by condition (VI), and for $N(s)$ the function $K \exp (K s)$, there exists a function $G$ corresponding to these choices with the properties (i) and (ii) above.

Define $V(t, x)=\int_{0}^{\infty} G(\|\Phi(t+s, t, x)\|) d s$.

The function $V$ has the following properties:

(V-i) $V \in C^{\prime}\left(I \times S_{R \mid x}\right)$.

(V-ii) Since $\|x\| \leqq|x|,\left|V_{x}(t, x)\right| \leqq \int_{0}^{\infty} G^{\prime}(d(R) \sigma(s)) K \exp (K s) d s \equiv P<\infty$ for all $(t, x) \in I \times S_{R \mid x}$.

(V-iii) $V(t, x) \leqq \int_{0}^{\infty} G(d(|x|) \sigma(s)) d s \equiv b_{V}(|x|)$ for all $(t, x) \in I \times S_{R \mid x}$.

(V-iv) Since $\|x\| \geqq|x| / k^{1 / 2}$ and $\|\Phi(t+s, t, x)\| \geqq\|x\| / 2$ for $0 \leqq s \leqq\|x\| / 2 K_{f}$,

$$
\begin{aligned}
V(t, x) & \geqq \int_{0}^{|x| / 2 K_{f} k^{1 / 2}} G(\|\Phi(t+s, t, x)\|) d s \\
& \geqq\left(|x| / 2 K_{f} k^{1 / 2}\right) G\left(|x| / 2 k^{1 / 2}\right) \equiv a_{V}(|x|)
\end{aligned}
$$

for $(t, x) \in I \times S_{R \mid x}$. Here $K_{f}$ denotes the upper bound of $f(t, x, 0,0)$ on $I \times S_{R \mid x}$.

$(\mathrm{V}-\mathrm{v})$ Since $V\left(t, \Phi\left(t, t_{0}, x_{0}\right)\right)=\int_{t}^{\infty} G\left(\left\|\Phi\left(s, t_{0}, x_{0}\right)\right\|\right) d s, d V\left(t, \Phi\left(t, t_{0}, x_{0}\right)\right) / d t$ $=-G\left(\left\|\Phi\left(t, t_{0}, x_{0}\right)\right\|\right)$ for all $\left(t_{0}, x_{0}\right) \in I \times S_{R \mid x}$ and $t \in I$. This fact is written as $V_{0}^{\prime}(t, x)=-G(\|x\|)$.

We observe that the functions $b_{V}$ and $a_{V}$ defined in (V-iii) and (V-iv) above are strictly increasing, continuous functions of $|x|$ with $a_{V}(0)=b_{V}(0)=0$. A function with the above properties $(\mathrm{V}-\mathrm{i})-(\mathrm{V}-\mathrm{v})$ is called a Liapunov function for the system (D).

The following lemma establishes the existence of a Liapunov function for the system (BL) which is, in a sense, uniform in the parameters $(\alpha, \beta) \in I \times S_{R \mid x}$.

LEMMA 2. Under the hypotheses of the theorem, there exists a Liapunov function $W(y, \alpha, \beta)$ for the system $(\mathrm{BL})$ for each $(\alpha, \beta) \in I \times S_{R \mid x}$ with the following properties:

(i) $W \in C^{\prime}\left(S_{R \mid y} \times I \times S_{R \mid x}\right)$.

(ii) There exists a constant $Q<\infty$ such that $\left|W_{y}(y, \alpha, \beta)\right| \leqq Q,\left|W_{\alpha}(y, \alpha, \beta)\right| \leqq Q$, and $\left|W_{\beta}(y, \alpha, \beta)\right| \leqq Q$ for all $(\alpha, \beta, y) \in I \times S_{R}$.

(iii) There exist continuous, strictly increasing functions $a_{W}(|y|)$ and $b_{W}(|y|)$ independent of $(\alpha, \beta)$ such that $a_{W}(0)=b_{W}(0)=0$ and $a_{W}(|y|) \leqq W(y, \alpha, \beta)$ $\leqq b_{W}(|y|)$ for all $(\alpha, \beta, y) \in I \times S_{R}$.

Proof of Lemma 2. The proof is accomplished by using Lemma 1. Let $\Psi\left(s, y_{0}, \alpha, \beta\right)$ denote the solution of system (BL). 
We first show that $\left|\Psi_{y}(s, y, \alpha, \beta)\right|$ is bounded above by a function of the form $K_{1} \exp \left(K_{1} s\right)$ for $0 \leqq s<\infty$ and $(\alpha, \beta, y) \in I \times S_{R}$ where $K_{1}$ is a constant independent of $(\alpha, \beta, y)$. This follows immediately from the boundedness of $g_{y}(\alpha, \beta, y, 0)$ for $(\alpha, \beta, y) \in I \times S_{R}$ and the fact that $\Psi_{y}(s, y, \alpha, \beta)$ is a fundamental matrix for the system

$$
d z / d s=g_{y}(\alpha, \beta, \Psi(s, y, \alpha, \beta), 0) z .
$$

Next, we see that similar estimates are valid for $\left|\Psi_{\alpha}(s, y, \alpha, \beta)\right|$ and $\left|\Psi_{\beta}(s, y, \alpha, \beta)\right|$. A well-known formula ([2,p. 25]) for the determinant of a fundamental matrix for a linear system gives

$$
\begin{aligned}
\operatorname{det} \Psi_{y}(s, y, \alpha, \beta) & =\exp \left\{\int_{0}^{s} \operatorname{tr} g_{y}(\alpha, \beta, \Psi(s, y, \alpha, \beta), 0) d s\right\} \\
& \geqq \exp \left\{-j K_{1} s\right\} .
\end{aligned}
$$

With this we can find a constant $K_{2}$ such that $\left|\Psi_{y}^{-1}(s, y, \alpha, \beta)\right| \leqq K_{2} e^{K_{2} S}$ for $(\alpha, \beta, y) \in I \times S_{R}$ and $0 \leqq s<\infty$. The function $\Psi_{\alpha}(s, y, \alpha, \beta)$ is a solution of the matrix differential equation

$$
d Z / d s=g_{y}(\alpha, \beta, \Psi(s, y, \alpha, \beta), 0) Z+g_{t}(\alpha, \beta, \Psi(s, y, \alpha, \beta), 0) .
$$

Applying the variation of constants formula gives

$$
\Psi_{\alpha}(s, y, \alpha, \beta)=\Psi_{y}(s, y, \alpha, \beta) \int_{0}^{s} \Psi_{y}^{-1}(u, y, \alpha, \beta) g_{t}(\alpha, \beta, \Psi(u, y, \alpha, \beta), 0) d u .
$$

Thus, we can find a constant $K_{3}$ which is independent of $(\alpha, \beta, y)$ such that

$$
\left|\Psi_{\alpha}(s, y, \alpha, \beta)\right| \leqq K_{3} \exp \left(K_{3} s\right)
$$

for $0 \leqq s<\infty$ and $(\alpha, \beta, y) \in I \times S_{R}$. A similar argument shows that we can find a constant $K_{4}$ independent of $(\alpha, \beta, y)$ such that $\left|\Psi_{\beta}(s, y, \alpha, \beta)\right| \leqq K_{4} \exp \left(K_{4} s\right)$ for $0 \leqq s<\infty$ and $(\alpha, \beta, y) \in I \times S_{R}$. Taking $\hat{K}$ to be the largest of $K_{1}, K_{2}, K_{3}$ and $K_{4}$, we have $\left|\Psi_{y}(s, y, \alpha, \beta)\right| \leqq \hat{K} \exp (\hat{K} s), \quad\left|\Psi_{\alpha}(s, y, \alpha, \beta)\right| \leqq \hat{K} \exp (\hat{K} s)$ and $\left|\Psi_{\beta}(s, y, \alpha, \beta)\right| \leqq \hat{K} \exp (\hat{K} s)$ for $0 \leqq s<\infty$ and $(\alpha, \beta, y) \in I \times S_{R}$.

For $y \neq 0$, the inequality

$$
|\partial\|\Psi(s, y, \alpha, \beta)\| / \partial y| \leqq\left|\Psi_{y}(s, y, \alpha, \beta)\right|,
$$

implies $|\partial||\Psi(s, y, \alpha, \beta) \|| \partial y \mid \leqq \hat{K} \exp (\hat{K} s)$ for $0 \leqq s<\infty$ and $(\alpha, \beta, y) \in I \times S_{R}$. Similarily, $|\partial\|\Psi(s, y, \alpha, \beta)\|| \partial \alpha \mid \leqq \hat{K} \exp (\hat{K} s)$ and $|\partial\|\Psi(s, y, \alpha, \beta)\| / \partial \beta| \leqq \hat{K} \exp (\hat{K} s)$ for $0 \leqq s<\infty,(\alpha, \beta, y) \in I \times S_{R}$ and $y \neq 0$.

If we take for $c$ and $\lambda$ in Lemma $1, e \in \mathscr{K}$ and $\rho \in \mathscr{S}$ given by condition (VII), and for $N(s)$ the function $\hat{K} \exp (\hat{K} s)$, there exists a function $H$ with the following properties. The functions $H(u)$ and $H^{\prime}(u)$ are continuous, strictly increasing functions for $u \geqq 0$ with $H(0)=H^{\prime}(0)=0$, and the integrals 


$$
\int_{0}^{\infty} H(u(s)) d s \text { and } \int_{0}^{\infty} H^{\prime}(u(s)) \hat{K} \exp (\hat{K} s) d s
$$

converge uniformly in $u$ for $0 \leqq u(s) \leqq e(R) \rho(s)$.

Define $W(y, \alpha, \beta)=\int_{0}^{\infty} H(\|\Psi(s, y, \alpha, \beta)\|) d s$.

It follows that $\mathrm{W}$ is the desired Liapunov function for the system (BL). Indeed, (W-i) $W \in C^{\prime}\left(S_{R \mid y} \times I \times S_{R \mid x}\right)$.

$\left(\mathrm{W}\right.$-ii) Let $Q=\int_{0}^{\infty} H^{\prime}(e(R) \rho(s)) \hat{K} \exp (\hat{K} s) d s$. Then, $\left|W_{y}(y, \alpha, \beta)\right| \leqq Q$, $\left|W_{\alpha}(y, \alpha, \beta)\right| \leqq Q$, and $\left|W_{\beta}(y, \alpha, \beta)\right| \leqq Q$ for all $(\alpha, \beta, y) \in I \times S_{R}$.

(W-iii) Let $a_{W}(|y|)=\left(|y| / 2 K_{g} j^{1 / 2}\right) H\left(|y| / 2 j^{1 / 2}\right)$ where $K_{g}$ is an upper bound for $g(t, x, y, 0)$ on $I \times S_{R}$. Then $W(y, \alpha, \beta) \geqq a_{W}(|y|)$ for all $(\alpha, \beta, y) \in I \times S_{R}$.

(W-iv) Let $b_{W}(|y|)=\int_{0}^{\infty} H(e(|y|) \rho(s)) d s$. Then, $W(y, \alpha, \beta) \leqq b_{W}(|y|)$ for all $(\alpha, \beta, y) \in I \times S_{R}$.

$(\mathrm{W}-\mathrm{v})$ Since

$$
\begin{aligned}
W\left(\Psi\left(s, y_{0}, \alpha, \beta\right), \alpha, \beta\right) & =\int_{0}^{\infty} H\left(\left\|\Psi\left(u, \Psi\left(s, y_{0}, \alpha, \beta\right), \alpha, \beta\right)\right\|\right) d u \\
& =\int_{s}^{\infty} H\left(\left\|\Psi\left(u, y_{0}, \alpha, \beta\right)\right\|\right) d u, \\
d W\left(\Psi\left(s, y_{0}, \alpha, \beta\right), \alpha, \beta\right) / d s & =-H\left(\left\|\Psi\left(s, y_{0}, \alpha, \beta\right)\right\|\right) .
\end{aligned}
$$

Clearly, $a_{W}$ and $b_{W}$ defined in (W-iii) and (W-iv) are strictly increasing, continuous functions with $a_{W}(0)=b_{W}(0)=0$.

This completes the proof of Lemma 2 . Let

and

$$
\begin{aligned}
l(c) & =\inf \{|x|: V(t, x)=c \text { for some } t \in I\}, \\
m(c) & =\sup \{|x|: V(t, x)=c \text { for some } t \in I\}, \\
l_{1}(\kappa) & =\inf \left\{|y|: W(y, t, x)=\kappa \text { for some }(t, x) \in I \times S_{R \mid x}\right\},
\end{aligned}
$$

$$
m_{1}(\kappa)=\sup \left\{|y|: W(y, t, x)=\kappa \text { for some }(t, x) \in I \times S_{R \mid x}\right\} .
$$

With these definitions it is clear that for $c$ and $\kappa$ sufficiently small, say $0<c<c^{0}$ and $0<\kappa<\kappa^{0}, 0<l(c) \leqq m(c)<\infty$ and $0<l_{1}(\kappa) \leqq m_{1}(\kappa)<\infty$. Also, $f m(c) \rightarrow 0$ as $c \rightarrow 0$ and $m_{1}(\kappa) \rightarrow 0$ as $\kappa \rightarrow 0$.

Lemma 2 is now applied to prove the following fundamental lemma.

Lemma 3. Let the hypotheses of the theorem be satisfied. Given any $c<c^{0}$, for sufficiently small $\kappa$ there exists $\varepsilon(c, \kappa) \leqq \varepsilon^{0}$ such that the "tube" described by

$$
\Gamma_{c, \kappa}=\left\{(t, x, y) \in I \times S_{R}: V(t, x) \leqq c \text { and } W(y, t, x) \leqq \kappa\right\}
$$

is invariant with respect to solutions of $\left(P_{\varepsilon}\right), 0<\varepsilon<\varepsilon(c, k)$. That is, any solution of $\left(P_{\varepsilon}\right), 0<\varepsilon<\varepsilon(c, \kappa)$, which meets $\Gamma_{c, \kappa}$ cannot leave it thereafter.

Proof of Lemma 3. The derivative of $V$ along solutions of $\left(P_{0}\right)$ is

$$
V_{0}^{\prime}(t, x)=V_{t}(t, x)+V_{x}(t, x) f(t, x, 0,0) \text {. }
$$


At the same time, $V_{0}{ }^{\prime}(t, x)=-G(\|x\|)$ (property V-v), so

$$
\begin{aligned}
V_{t}(t, x)+V_{x}(t, x) \cdot f(t, x, 0,0) & =-G(\|x\|) \\
& \leqq-G\left(|x| / k^{1 / 2}\right) .
\end{aligned}
$$

Combining this with the boundedness of $\left|V_{x}(t, x)\right|$ on $I \times S_{R \mid x}$ and smoothness condition (IV) gives the following estimate for the derivative of $V(t, x)$ along solutions of $\left(P_{\varepsilon}\right)$ :

$$
\begin{aligned}
V_{\varepsilon}^{\prime}(t, x) & =V_{t}(t, x)+V_{x}(t, x) \cdot f(t, x, y, \varepsilon) \\
& =V_{0}^{\prime}(t, x)+V_{x}(t, x)[f(t, x, y, \varepsilon)-f(t, x, 0,0)] \\
& \leqq-G\left(|x| / k^{1 / 2}\right)+P v(|y|, \varepsilon)
\end{aligned}
$$

for $(t, x, y) \in I \times S_{R}$ and $0<\varepsilon \leqq \varepsilon^{0}$.

Similarly, we obtain an estimate for the derivative of $W$ along the solutions of $\left(P_{\varepsilon}\right)$. The derivative of $W$ along the solutions of $\left(P_{\varepsilon}\right)$ is

$$
\begin{aligned}
W_{\varepsilon}^{\prime}(y, t, x)= & (1 / \varepsilon) W_{y}(y, t, x) \cdot g(t, x, y, \varepsilon)+W_{t}(y, t, x) \\
& +W_{x}(y, t, x) \cdot f(t, x, y, \varepsilon) .
\end{aligned}
$$

The derivative of $W$ along the solutions of (BL) is

$$
d W(y, t, x) / d s=W_{y}(y, t, x) \cdot g(t, x, y, 0) .
$$

At the same time, $d W(y, t, x) / d s=-H(\|y\|) \leqq-H\left(|y| / j^{1 / 2}\right)$ (property W-v). Thus,

$$
W_{y}(y, t, x) \cdot g(t, x, y, 0) \leqq-H\left(|y| / j^{1 / 2}\right)
$$

for $(t, x, y) \in I \times S_{R}$. Combination of these remarks with property (W-ii) and smoothness conditions (IV) and (V) gives

$$
\begin{aligned}
W_{\varepsilon}^{\prime}(y, t, x)= & (1 / \varepsilon)\left\{W_{y}(y, t, x) \cdot g(t, x, y, 0)\right. \\
& \left.+W_{y}(y, t, x) \cdot[g(t, x, y, \varepsilon)-g(t, x, y, 0)]\right\} \\
& +W_{\iota}(y, t, x)+W_{x}(y, t, x) \cdot f(t, x, 0,0) \\
& +W_{x}(y, t, x) \cdot[f(t, x, y, \varepsilon)-f(t, x, 0,0)] \\
\leqq & (1 / \varepsilon)\left\{-H\left(|y| / j^{1 / 2}\right)+Q \mu(\varepsilon)\right\}+Q\left[1+K_{f}+v(|y|, \varepsilon)\right]
\end{aligned}
$$

for $(t, x, y) \in I \times S_{R}$ and $0<\varepsilon \leqq \varepsilon^{0}$.

The boundary of the set

$$
\Gamma_{c, \kappa}=\left\{(t, x, y) \in I \times S_{R}: V(t, x) \leqq c \text { and } W(y, t, x) \leqq \kappa\right\}
$$


is the union of two sets:

and

$$
\Gamma_{1}=\left\{(t, x, y) \in I \times S_{R}: V(t, x)=c \text { and } W(y, t, x) \leqq \kappa\right\},
$$

$$
\Gamma_{2}=\left\{(t, x, y) \in I \times S_{R}: V(t, x) \leqq c \text { and } W(y, t, x)=\kappa\right\} .
$$

Given $c, 0<c<c^{0}$, we shall find $\kappa(c), 0<\kappa(c)<c$, with the property that for each $\kappa, 0<\kappa \leqq \kappa(c)$, there exists $\varepsilon(c, \kappa), 0<\varepsilon(c, \kappa)<\varepsilon^{0}$, such that $V_{\varepsilon}^{\prime}$ is negative on $\Gamma_{1}$ and $W_{\varepsilon}^{\prime}$ is negative on $\Gamma_{2}$ provided $0<\varepsilon<\varepsilon(c, \kappa)$.

Indeed, for $(t, x, y) \in \Gamma_{1},|x| \geqq l(c)$ and $a_{W}(|y|) \leqq \kappa$. Let us choose $0<\kappa(c)$ $<\min \left(\kappa^{0}, c\right)$ and $0<\varepsilon^{\prime} \leqq \varepsilon^{0}$ such that $a_{W}(|y|) \leqq \kappa(c)$ and $0<\varepsilon<\varepsilon^{\prime}$ imply $v(|y|, \varepsilon)<G\left(l(c) / k^{1 / 2}\right) / P$. It then follows from(11) that $V_{\varepsilon}^{\prime}(t, x)<0$ for $(t, x, y)$ $\in \Gamma_{1}$ provided $0<\varepsilon<\varepsilon^{\prime}$ and $0<\kappa<\kappa(c)$.

For $(t, x, y) \in \Gamma_{2},|y| \geqq l_{1}(\kappa)$. Let us choose $0<\varepsilon(c, \kappa)<\varepsilon^{\prime}$ such that $\mu(\varepsilon)<H\left[l_{1}(\kappa) / j^{1 / 2}\right] / 2 Q$ and

$$
1 / \varepsilon>2 Q\left[1+\kappa_{f}+(G(R) / P)\right] / H\left[l_{1}(\kappa) / j^{1 / 2}\right]
$$

for $0<\varepsilon<\varepsilon(c, \kappa)$. It follows from (12) that $W_{\varepsilon}{ }^{\prime}<0$ on $\Gamma_{2}$ for $\varepsilon<\varepsilon(c, \kappa)$.

From this it is clear that any solution of $\left(P_{\varepsilon}\right), 0<\varepsilon<\varepsilon(c, \kappa)$, which meets the boundary of $\Gamma_{c, \kappa}, 0<\kappa<\kappa(c)$, proceeds into the interior.

This establishes Lemma 3.

The existence of solutions of $\left(P_{\varepsilon}\right)$ for sufficiently small $\varepsilon$ and $\left|x_{0}\right|+\left|y_{0}\right|$ follows immediately from Lemma 3. Take $c_{1}, 0<\kappa_{1} \leqq \kappa\left(c_{1}\right)$, and $0<\varepsilon_{1}=\varepsilon\left(c_{1}, \kappa_{1}\right)$ such that the tube $\Gamma_{c_{1}, \kappa_{1}}$ lies in the interior of $I \times S_{R}$ and is invariant with respect to solutions of $\left(P_{\varepsilon}\right), 0<\varepsilon<\varepsilon_{1}$. It then follows that solutions of $\left(P_{\varepsilon}\right), 0<\varepsilon<\varepsilon_{1}$, whose initial-values lie in $D=\left\{(x, y) \in S_{R}: V\left(t_{0}, x\right) \leqq c_{1}\right.$ and $\left.W\left(y, t_{0}, x\right) \leqq \kappa_{1}\right\}$ exist for $t_{0} \leqq t<\infty$.

The following lemma was first proved by A. N. Tihonov [8]. A proof is presented here for two reasons: The proof gives some insight into the behavior of the solutions of $\left(P_{\varepsilon}\right)$ for $t$ near $t_{0}$, and Lemma 3 provides a simple proof of this lemma.

LEMMA 4. Under the hypotheses of the theorem, for any $\left(x_{0}, y_{0}\right) \in D$ the solution of $\left(P_{\varepsilon}\right)$ tends to the solution of $\left(P_{0}\right)$ as $\varepsilon \rightarrow 0^{+}$uniformly on any set of the form $t_{0}<t_{1} \leqq t \leqq T$.

Proof of Lemma 4. Let $x=\phi(t, \varepsilon), y=\psi(t, \varepsilon)$ be the solution of $\left(P_{\varepsilon}\right)$ for some $\left(x_{0}, y_{0}\right) \in D$. By the remark above, this solution exists for $t_{0} \leqq t \leqq T$ provided $0<\varepsilon<\varepsilon_{1}$.

Let us first show that $|\psi(t, \varepsilon)| \rightarrow 0$ as $\varepsilon \rightarrow 0^{+}$uniformly for $t_{1} \leqq t \leqq T$. That is, for any $\gamma>0$ we must find $\varepsilon(\gamma) \leqq \varepsilon_{1}$ such that $|\psi(t, \varepsilon)|<\gamma$ for $t_{1} \leqq t \leqq T$ provided $0<\varepsilon<\varepsilon(\gamma)$. Taking $\kappa(\gamma)<\kappa_{1}$ such that $|y|<\gamma$ for $a_{W}(|y|) \leqq \kappa(\gamma)$, we apply Lemma 3 to obtain $\varepsilon\left(c_{1}, \kappa(\gamma)\right)$ such that any solution of $\left(P_{\varepsilon}\right), 0<\varepsilon<\varepsilon\left(c_{1}, \kappa(\gamma)\right)$, which meets the set $\Gamma_{c_{1}, \kappa(\gamma)}$ cannot escape. It is thus sufficient to show that for sufficiently small $\varepsilon,\left(t_{1}, \phi\left(t_{1}, \varepsilon\right), \psi\left(t_{1}, \varepsilon\right)\right) \in \Gamma_{c_{1}, \kappa(\gamma)}$. 
Take $\theta(\gamma)>0$ such that $|y| \leqq \theta(\gamma)$ implies $b_{W}(|y|) \leqq \kappa(\gamma)$. From Condition (VII) we have that $\left|\Psi\left(s, y_{0}, t_{0}, x_{0}\right)\right| \rightarrow 0$ as $s \rightarrow \infty$, so there exists $s_{1}>0$ such that $s_{1} \leqq s<\infty$ implies $\left|\Psi\left(s, y_{0}, t_{0}, x_{0}\right)\right|<\theta(\gamma) / 2$. Let $x=\phi_{1}(s, \varepsilon), y=\psi_{1}(s, \varepsilon)$ be be the solution of

$$
\begin{array}{ll}
d x / d s=\varepsilon f\left(t_{0}+\varepsilon s, x, y, \varepsilon\right), & x(0)=x_{0}, \\
d y / d s=g\left(t_{0}+\varepsilon s, x, y, \varepsilon\right), & y(0)=y_{0} .
\end{array}
$$

We see from this system that $\psi_{1}(s, \varepsilon) \rightarrow \Psi\left(s, y_{0}, t_{0}, x_{0}\right)$ as $\varepsilon \rightarrow 0^{+}$for each $0 \leqq s<\infty$. Thus, there exists $\varepsilon_{2}<\min \left\{\left(t_{1}-t_{0}\right) / s_{1}, \varepsilon_{1}\right\}$ such that $\left|\psi_{1}\left(s_{1}, \varepsilon\right)-\Psi\left(s_{1}, y_{0}, t_{0}, x_{0}\right)\right|$ $<\theta(\gamma) / 2$ for $0<\varepsilon<\varepsilon_{2}$. Since $\psi(t, \varepsilon)=\psi_{1}\left(\left(t-t_{0}\right) / \varepsilon, \varepsilon\right), \quad|\psi(t, \varepsilon)|<\theta(\gamma)$ for $t=t_{0}+\varepsilon s_{1}$ and $0<\varepsilon<\varepsilon_{2}$. Also, $\varepsilon_{2}<\left(t_{1}-t_{0}\right) / s_{1}$ implies $t_{0}<t_{0}+\varepsilon s_{1}<t_{1}$ for $0<\varepsilon<\varepsilon_{2}$. Thus, for each $0<\varepsilon<\varepsilon_{2}, W(\psi(t, \varepsilon), t, x) \leqq b_{W}(|\psi(t, \varepsilon)|) \leqq \kappa(\gamma)$ for $t=t_{0}+\varepsilon s_{1}$; i.e., $\left(t_{0}+\varepsilon s_{1}, \phi\left(t_{0}+\varepsilon s_{1}, \varepsilon\right), \psi\left(t_{0}+\varepsilon s_{1}, \varepsilon\right)\right) \in \Gamma_{c_{1}, \kappa(\gamma)}$ for each $0<\varepsilon$ $<\varepsilon_{2}$. Hence, by Lemma $3, a_{W}(|\psi(t, \varepsilon)|) \leqq W(\psi(t, \varepsilon), t, x) \leqq \kappa(\gamma)$ for $t_{1} \leqq t \leqq T$ provided $0<\varepsilon<\min \left\{\varepsilon_{2}, \varepsilon\left(c_{1}, \kappa(\gamma)\right)\right\} \equiv \varepsilon(\gamma)$.

It remains to show that $\phi(t, \varepsilon)$ converges to the solution of $(D)$ as $\varepsilon \rightarrow 0^{+}$uniformly for $t_{1} \leqq t \leqq T$. This is accomplished by observing that $x=\phi(t, \varepsilon)$ satisfies

$$
x^{\prime}=f(t, x, \psi(t, \varepsilon), \varepsilon), \quad x\left(t_{0}+\varepsilon s_{1}, \varepsilon\right)=x_{0}+\delta(\varepsilon)
$$

where $\delta(\varepsilon) \rightarrow 0$ as $\varepsilon \rightarrow 0^{+}$. By a well-known theorem on continuous dependence of solutions on a parameter and on initial conditions [2, p. 29], it follows that $\phi(t, \varepsilon) \rightarrow \Phi(t)$, the solution of (D), as $\varepsilon \rightarrow 0^{+}$uniformly on $t_{1} \leqq t \leqq T$.

This completes the proof of Lemma 4.

The proof of the theorem is now easily completed. Given any $\eta>0$ and $t_{1}>t_{0}$, we must verify that there exists an $\varepsilon(\eta)>0$ such that $|\phi(t, \varepsilon)-\Phi(t)|+|\psi(t, \varepsilon)|<\eta$ for $t_{1} \leqq t<\infty$ and $0<\varepsilon<\varepsilon(\eta)$. Here again, $x=\phi(t, \varepsilon), y=\psi(t, \varepsilon)$ denotes the solution of $\left(P_{\varepsilon}\right)$, and $x=\Phi(t), y=0$ denotes the solution of $\left(P_{0}\right)$.

Choose $c_{2}>0$ such that $\Gamma_{c_{2}, \kappa\left(c_{2}\right)} \subset I \times S_{\eta / 3}$ where $\kappa\left(c_{2}\right)$ is given by Lemma 3 . Furthermore, choose $0<r<\eta / 3$ such that $|x| \leqq r$ implies $b_{V}(|x|) \leqq c_{2}$ and $|y| \leqq r$ implies $b_{W}(|y|) \leqq \kappa\left(c_{2}\right)$.

Condition (VI) implies $\Phi(t) \rightarrow 0$ as $t \rightarrow \infty$, hence there exists $T_{\eta}>t_{1}$ such that $|\Phi(t)|<r / 2$ for $t \geqq T_{\eta}$. Lemma 4 provides an $\varepsilon(r)>0$ such that $0<\varepsilon<\varepsilon(r)$ implies $|\phi(t, \varepsilon)-\Phi(t)|+|\psi(t, \varepsilon)|<r / 2$ for $t_{1} \leqq t \leqq T_{\eta}$. Thus, $|\phi(t, \varepsilon)|+|\psi(t, \varepsilon)|$ $\leqq|\phi(t, \varepsilon)-\Phi(t)|+|\Phi(t)|+|\psi(t, \varepsilon)|$ implies $\left|\phi\left(T_{\eta}, \varepsilon\right)\right|+\left|\psi\left(T_{\eta}, \varepsilon\right)\right|<r$ for $0<\varepsilon<\varepsilon(r)$. That is, $\left(T_{\eta}, \phi\left(T_{\eta}, \varepsilon\right), \psi\left(T_{\eta}, \varepsilon\right)\right) \in \Gamma_{c_{2}, \kappa\left(c_{2}\right)}$ for $0<\varepsilon<\varepsilon(r)$. Lemma 3 implies that $\phi(t, \varepsilon), \psi(t, \varepsilon)$ remains in $\Gamma_{\left.c_{2}, \kappa^{\prime} c_{2}\right)}$ for $t \geqq T_{\eta}$ provided $0<\varepsilon<\varepsilon\left(c_{2}, K\left(c_{2}\right)\right)$.

Thus, it follows that $|\phi(t, \varepsilon)-\Phi(t)|+|\psi(t, \varepsilon)|<\eta$ for $t_{1} \leqq t<\infty$ provided $0<\varepsilon<\min \left\{\varepsilon(r), \varepsilon\left(c_{2}, \kappa\left(c_{2}\right)\right)\right\}$.

This completes the proof. 


\section{BIBLIOGRAPHY}

1. V. F. Butuzov, Asymptotic formulae for solutions of systems of differential equations with small parameter at the derivative in the semi-infinite interval, Vestnik Moscov. Univ. 4 (1963), 3-14. (Russian)

2. E. A. Coddington and N. Levinson, Theory of ordinary differential equations, McGrawHill, New York, 1955.

3. L. Flatto and N. Levinson, Periodic solutions of singularly perturbed systems, J. Rational Mech. Anal. 4 (1955), 943-950.

4. K. O. Friedrichs and W. Wasow, Singular perturbations of non-linear oscillations, Duke Math. J. 13 (1946), 367-381.

5. J. J. Levin and N. Levinson, Singular perturbations of non-linear systems of differential equations and associated boundary layer equation, J. Rational Mech. Anal. 3 (1954), 247-270.

6. W. Hahn, On the general concept of stability and Liapunov's direct method, Technical Rep. No. 485, U.S. Army Mathematics Research Center, Madison, Wisconsin, 1964.

7. J. L. Massera, On Liapunov's conditions of stability, Ann. of Math. 50 (1949), 705-721.

8. A. N. Tihonov, Systems of differential equations containing a small parameter multiplying the derivative, Mat. Sb. N. S. (31) 73 (1952), 575-585. (Russian)

\section{Michigan State University,}

EAST LANSING, Michigan 